\title{
Methodology For Modeling Rural Development - Case Study From The Western Balkans
}

\section{Sofija Adzic}

\begin{abstract}
This paper tries to find more efficient approach to defining the attributes and goals of rural development policy. The proposed methodology for defining the future role of rural regions within the socio-economic framework of the Western Balkans enables a very precise and transparent definition of rural development goals.
\end{abstract}

JEL: R11

\section{Introduction}

Current social and economic trends coupled with the low controllability of socio-economic systems in the Western Balkan countries suggest the need to look for more efficient approaches to defining the attributes and goals of rural development policy. Discord between the theoretical basis and practical policy of rural development largely arises from: (1) intensive changes to the elements and structure of feed-backs in the socio-economic systems of each state in the Western Balkans during the process of transition from socialistic to capitalistic stimuli and limitations, (2) the poor quality of information due to the deformed system of social values and the domination of the interests of political elites and coalitions oriented to distribution and (3) the structure of interests wherein, with great difficulty and very slowly, solutions for overcoming socioeconomic conflicts and problems are articulated.

For these reasons, the author proposes a combined methodology for modeling rural development on the basis of (1) a precise definition of the future role of rural regions within the socio-economic framework of Western Balkan countries and (2) treating the problem of the definition of rural policies in the Western Balkans as one essentially of structure. The proposed methodology for defining the future role of rural regions within the socio-economic framework of the Western Balkans enables
DOI: $10.2478 / v 10033-007-0018-9$

a very precise and transparent definition of rural development goals.

Treating the problem of defining rural policy as a structural problem allows (1) the application of a methodology for soft systems and (2) the concept of decision-making on the basis of a layered, functional hierarchy and, in turn, the efficient integration of different ideas and proposals concerning more specific problems facing the rural regions of the Western Balkans. Because modeling rural policies is primarily based on verbal information, the methodology for the analysis of decision-making is based partly on fuzzy logic.

The basic goals of the proposed methodology are: (1) creating a mathematically exact model of rural development to simulate the costs and effects of individual decisions on rural policies, (2) the efficient and systematic use of the paradigms of competition, cooperation and learning as a response to the widespread institutional non-regulation characteristic of the Western Balkans (3) reaffirming the importance of regional cooperation and discourse in reaching a common, productive conception of the future role of rural regions within the Western Balkans and the policy that would best help realize this.

*Adzic: Faculty of Economics in Subotica

Segedinski put 9-11, Yu 24000 Subotica, Serbia

e-mail: adzic@eccf.su.ac.yu 


\section{Basic Methodology For the Precise Definition of the Future Role of Rural Regions Within a Larger Socio- economical Framework}

\subsection{Introduction}

Designing the goals and actions of the development of rural regions in the Western Balkans requires the consideration of complex political, scientific and pragmatic factors. A precise and transparent definition of the goals for the development of rural regions in the Western Balkans is indispensable to making decisions or devising strategies on the social, organizational, group or individual level. The future social or economic development- or transformation- of a rural region should arise from the basic question "What should rural settlements represent in the future?" In the past, imaginary, hypocritical and unscientific definitions of development strategy were the basic reasons that nearly two centuries after their independence from the Ottoman and Austrian Empires, the majority of rural regions in the Western Balkans were far behind their European counterparts in terms of structural adaptation and modernization.

The search for an answer to the question stated above is necessary for the future definition of the goals and actions of rural policy. The uncertainty in forecasting the condition of rural regions in the Western Balkans represents the principal challenge and often causes political and intellectual elites to abandon this task.

This work is an attempt to define the methodology for the definition of the future role of rural regions in the Western Balkans within a larger socio-economic framework, and in doing so to provide the basis for a scientific, precise, transparent and active rural policy. Its thesis is that the search for the new role of rural regions within the socio-economic framework of the Western Balkans is necessary in defining a strategy for rural development. The basic premises of the proposed methodology are:

First, precisely, transparently and clearly defined general socio-economic orientations substantially define rural development.

Second, a methodology for the definition of the goals and actions of rural policy should, on the one hand, accurately, clearly and transparently define these goals and, on the other, introduce sufficiently robust mechanisms for their realization in adverse situations, including those without a tradition of formal structures or previous policy intervention.

Third, that within the observed period of time, two or more competitive socio-economic orientations for the general development of rural regions cannot coexist; rather a process occurs that is characterized by the recession of one orientation followed by the implementation of a second.

Fourth, rural development is synonymous with the expansion of general socio-economic orientations into the entire rural socio-economic system, with the internal, rural economy serving as the basis for socio-economic development (adhering, inter alia, with the traditional rural culture of the Western Balkans).

Fifth, the forecast of rural development is the expression in terms of probability of the chances for a given socio-economic orientation (either a priori or anticipated) to become dominant over a period of time specified in advance.

Sixth, when conceiving of the future role of rural regions within the socio-economic development of the Western Balkans, this work explicitly forecasts a high level of cooperation between countries in terms of knowledge and experience exchange within the framework of their joint preparation for European integration processes. Special importance should be placed on the critical analysis of the goals, mechanisms and results of rural development in Greece, the sole country from this area with both actual experience of internal adaptation to European criteria and similarities in its political, social, economic and cultural systems of value to those of nations in the Western Balkans.

\subsection{Theoretical bases of the methodology for the precise definition of the future socio-economic orientation of rural regions}

The methodology presented here for the precise definition of the future socio-economic orientations of rural regions in the Western Balkans is based on the following presumptions:

First, the generation of new rural socio-economic orientations for rural regions is a complex event that can only be realized through the simultaneous actions of a large number of factors.

Second, the development of each of those factors is a 
random process - for this reason, the probability of the appearance of a foreseen situation at a given time $t$ (or at the period of time $\Delta \mathrm{t}$ ) represents basic information for the forecast of rural development.

Third, the factors are independent of one another, meaning that the generation of a (new) general socio-economic orientation is a complex event created by the simultaneous realization of some number of favorable, simple and mutually independent events. The favorable simple event is defined as a state of individual factors which does not prevent the generation of a new socio-economic orientation.

Fourth, the basic factors - generators of the creation of a new socio-economic orientation - are: (1) rural social and economic problems which cannot be solved within the framework of the ruling socio-economic relations - $\mathrm{P}_{\mathrm{RSE}^{\prime}}$ (2) knowledge needed for the development of new socio-economic relations to solve rural socio-economic problems - $\mathrm{K}_{\text {RSEP, }}$ (3) development needs characterizing (or which will characterize) the socio-economic or more general development of rural regions in the period of time for which the definition of socio-economic development is made $-\mathrm{D}_{\text {RSEN⿱}}$ (4) criteria expressed by the prevailing system of values (in terms of new rural socio-economic and cultural values) - KRSEV and (5) conditions expressing the (un)suitability of the economic, institutional and cultural aspects of rural infrastructure to accept the new rules of the game (new socio-economic orientations in terms of new socio-economic cultural values) $-C_{\text {RSEB }}$.

Fifth, the generation of new socio-economic orientations concerning the social and economic roles of rural regions will only occur when all five factors specified above have been realized. Because it is very difficult to define when exactly these necessary factors have been realized, and because within each some allowance should be made for what would constitute their realization, it is possible to substitute different levels of

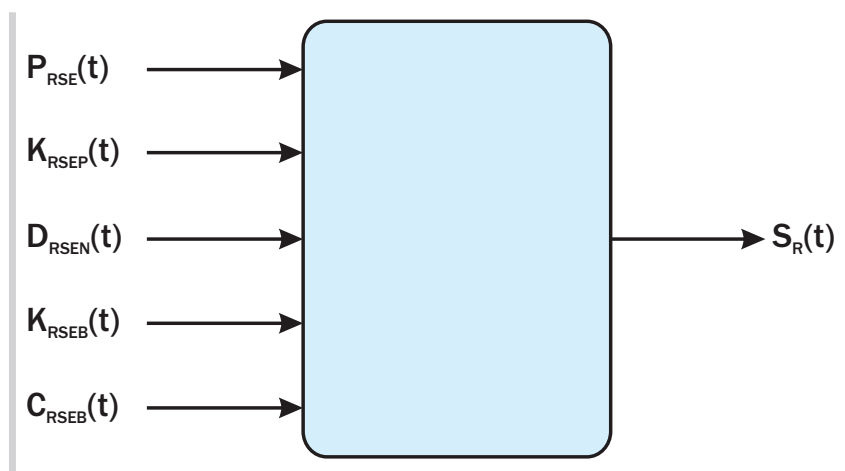

Figure 2.2.1.

System for the precise definition of the general rural socio-economic orientation of the future roles of rural regions. suitability, with the final judgment on the chances for the generation of new socio-economic orientations being made on the basis of the probability of a complex event. A new socioeconomic orientation for the future roles of rural regions in the Western Balkans is treated in the present work as an output from a system consisting of the inputs of the five factors specified above (which may be, in part, mutually substituted) (Figure 2.2.1).

The following is an outline of the procedure for foreseeing the states of the five specified factors:

Rural social and economic problems - $\mathrm{P}_{\mathrm{RSE}}$. Every prevailing socio-economic orientation solves some number and type of developmental, social or economic problems. At the same time, however, it creates new ones (social, economic, inter alia) which cannot be solved by means of the given orientation. This is a natural feature of each socio-economic orientation and is a basic motivation for the development of new (socioeconomic) orientations that can solve the problems created by the prevailing socio-economic orientation. The intensity of the need for new socio-economic orientation grows alongside the social and economic problems created by the prevailing socioeconomic orientation.

In our concrete case these include socialistic industrialization, the suppression of private initiatives and the neglect of rural regions. Development in the Western Balkans generated the following basic social and economic problems: (1) the demographic disruption of rural settlements in depressed hilly/mountainous and border regions, (2) aggravation of the age structure of rural populations, (3) very low standards of living relative to surrounding areas and (4) the immobilization of enormous natural and manmade resources. The growing severity of these problems signaled the necessity of a new socio-economic orientation. However, a high level of realism is required, for the necessity is not expressed in an explicit way, i.e., in the form of clear political, social and economic goals adapted to concrete needs and resources.

A compulsory transition (this "compulsion" should not be understood in its traditional form - i.e. imposed by external factors - but as the combination of such factors as international trade, social upheaval and indistinct changes to traditional living and working patterns from the influence of western civilization) led to a sudden and radical destruction of the former system and subsequent attempts to reform it.

In the Western Balkans, no attempt was made to develop or even define new socio-economic goals; goals from the wider environment that were often inappropriate for the region 
were simply accepted. Without analyzing these here, it may be noted that their actual implementation in the Western Balkans was relatively unsuccessful, at least in terms of intensifying social and economic development. In fact, during the first phase of transition, rural regions were more affected by the development of new systems of social and economic values than urban ones. Here we will simply try to formally define the probability of a set of problems most conducive to the appearance of new socio-economic orientations.

Theoretically, the range of rural social and economic problems created by prevailing socio-economic relations falls between the following two conditions: (1) existing socio-economic relations do not (yet) create insoluble social and economic problems and (2) social and economic problems are irreversible and beyond the aid of the existing social-economic relations.

Accordingly, the probability $\left(\mathrm{P}_{\mathrm{r}}\right)$ of the generation of such a state of social and economic problems which is suitable for the appearance of new socio-economic orientations - $\mathrm{P}_{\mathrm{r}}\left(\mathrm{P}_{\mathrm{RSE}}\right)$ is a function of the intensity of needs for new socio-economic relations.

The second factor needed for forming socio-economic orientations that will revive rural social and economic development is knowledge (for solving rural socio-economic problems) - $\mathrm{K}_{\mathrm{RSEP}}$. This knowledge for the solution to a given concrete rural socio-economic problem consists of three variables:

- $\mathrm{K}_{\text {RSEP }}(1)$ - immediately accessible knowledge

- $\quad \mathrm{K}_{\mathrm{RSEP}}(2)$ - knowledge accessible through research

- $\quad \mathrm{K}_{\mathrm{RSEP}}(3)$ - knowledge inaccessible because beyond socioeconomic and natural limitations.

New socio-economic orientations in terms of precise knowledge of new rural social and economic needs and the means for their provision (thereafter referred to as SRD) can be represented by:

\section{$S_{R D}=K_{R S E P}(1) \cup K_{\text {RSEP }}(2)$}

-provided that $\mathrm{K}_{\mathrm{RSEP}}(2)$ is empty. The probability that $\operatorname{Pr}\left(\mathrm{K}_{\mathrm{RSEP}}(2)\right)$, at a moment $t$, will be empty (i.e., that all necessary knowledge for the solution of rural social and economic problems will be available) is the relation between the variable $\mathrm{K}_{\mathrm{RSEP}}(1)$ and the union of the variables $K_{\text {RSEP }}(1) \cup K_{\text {RSEP }}(2)$, provided that $K_{\text {RSEP }}(2)$ is empty.

The developmental (social and economic) needs of rural regions - $\mathrm{P}_{r}\left(\mathrm{~K}_{\mathrm{RSEP}}(2)\right)$ are defined on the basis of the assumption that those with the best chance of becoming general socioeconomic orientations are most in harmony with the natural trajectory of the progression of basic human social, economic and cultural needs that can be met with a relevant conception of rural policy. Over this period of time, there are several needs which should be solved by means of rural social and economic policies.

Those needs should be defined from by the basic forms of the societalsocial and economical problems of rural regions in theof Western Balkans countries, specified above: (1) Ddemographic emptyingdisruptions of rural settlements in depressed, hilly-mountainous and border regions, (2) Aggravationchanges to of the age structure of rural populations in rural settlements, (3) Vvery low standards of living relativecompared with relevant to surrounding areas and (4) limmobilization of enormous natural and manmadeby work created resources.

These problems react differently to the appearance of new socio-economic orientations with set goals for policy in rural regions. For example, if an emphasis is placed on halting the depopulation in depressed hilly-mountainous and border regions, then the primary developmental, social and economic need should be the activation of natural and manmade resources. The second typical situation is the analysis of the development of social and economic needs removed temporally from its original situation; for example, improving the standard of living in rural regions to that of more dynamic urban ones. The probability $\left(\mathrm{P}_{\mathrm{r}}\right)$ that a rural social or economic need will benefit from the appearance of new socio-economic orientations - $P_{r}\left(D_{\text {RSEN }}\right)$ - is proportional to the conformity of this rural social or economic need to the trajectory of natural development needs.

The selection of criteria expressed by the prevailingrulings system of values - (in terms of (new) rural societalsocial, and economical and(cultural) values) - $\mathrm{K}_{\mathrm{RSEV}}$, in order to define the hierarchy of priorities of societalsocial-economical needs for the definition of goals and actions of rural policy is the reflectxion of the ratio of might between key economical and political groups. Consequently, the probability $\left(\mathrm{P}_{\mathrm{r}}\right)$ of the action of criteria in defining the priorities of societalsocial-economic al needs and, in turn,for the definition of the goals and actions of rural policy, , in conformity with positive changes in rural regions is proportional to their conformityadherence to with the priorities of key economical and political groups (being, inat this moment, based on their needsdesire to gain maximum profit and positions in the distribution of societalsocial wealth and influencemight overin the framework of existing resources - even when this diminishes these same resourcescauses their 
decrease as a whole!).

The political, institutional, social and economic infrastructure (the new socio-economic orientations in terms of new social, economic and cultural values) $C_{\text {RSEB }}$ is the last factor included in our attempt at modeling rural policy. The essence of this factor involves the following: the perceptions and interests of the political elite; transparency in the work of political institutions and organizations; the quality of legislative and administrative institutions and organizations and efficiency in decision making; development level, structure, location and programme orientation of innovative potential; level of development, structure and quality of education systems; structure allocation and efficiency of the network of social institutions and organizations; national structure of economy; structure of the market of supply and demand; quality and degree of development of trade, traffic and financial organizations; and investment capability. All of these - given a socio-economic orientation that formulates the new goals and actions of rural policy - may exist in harmony or, more frequently, in conflict to a greater or lesser degree. This is the most important factor for development and implementation. The non-conformity of this factor at the request of a particular socio-economic interest might wholly prevent its implementation. Consequently, the probability $\left(\mathrm{P}_{\mathrm{r}}\right)$ of the effect of the entire national political, institutional, social and economic infrastructure relative to its definition within a hierarchy of socio-economic needs according to the goals and actions of the given rural policy is proportional to its conformity to the development and priorities of this factor.

\subsection{Structure of the methodology for the definition of socio-economic orientations for the future roles of rural regions}

It has been demonstrated above that the formation of new socio-economic orientations in order to define the goals and actions of a rural policy requires the following: (1) Significant social and economic problems in rural regions within the framework of existing socio-economic relations demanding immediate solution, (2) the necessary knowledge for the solution of rural social and economic problems, (3) the necessary level of concordance between relevant socio-economic orientations (and their acknowledgement) and the need for development, (4) Sufficient consent between socio-economic orientations for overcoming socio-economic problems in rural regions and the prevailing systems of criteria and values (5) sufficient level of development of political, institutional, social and economic infrastructure for the implementation of new socio-

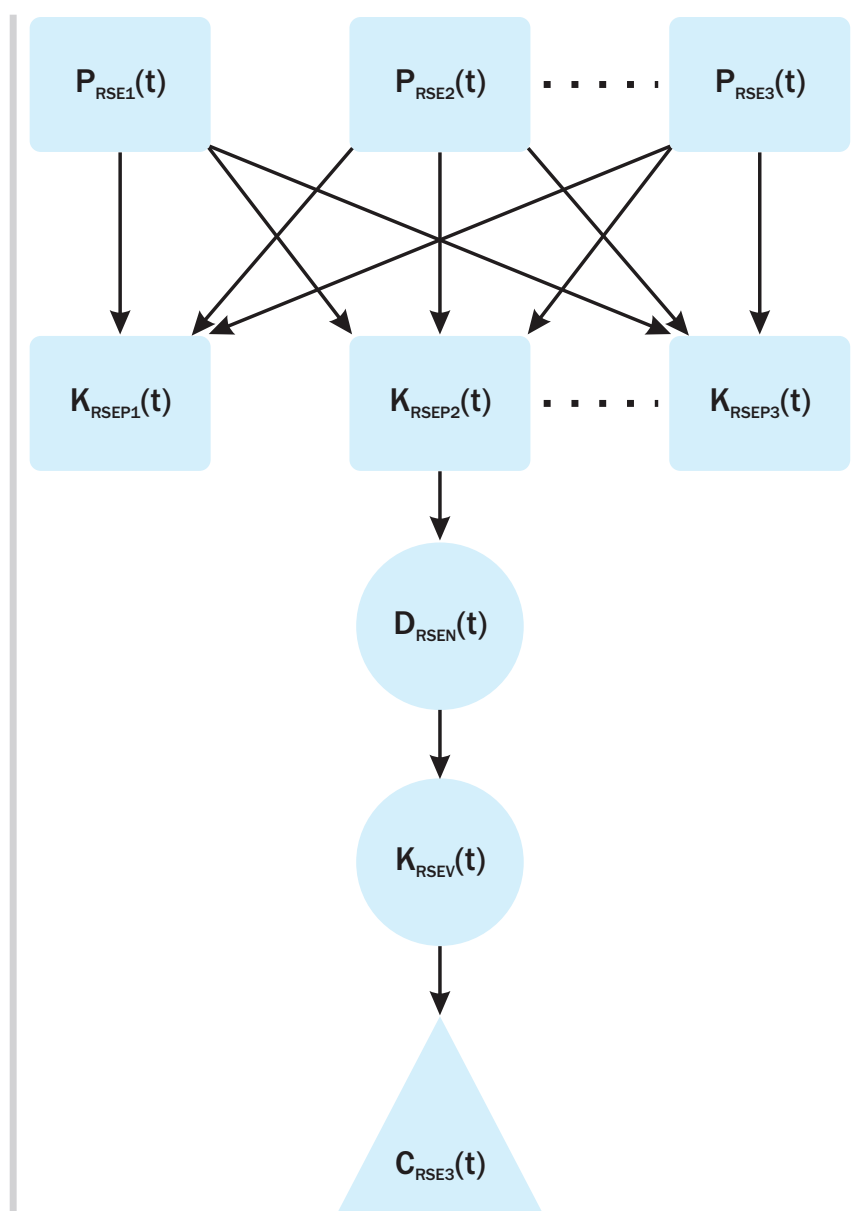

Figure 2.3.1.

The analytical procedure for the definition of general socio-economic orientations of the future roles of rural regions

economic orientations in the domain of rural development. In the methodology outlined in this study, we will introduce the following limitations.

We are going to suppose that over this period of time, there are several rural socio-economic problems: $P_{\text {RSE1 }}, P_{\text {RSE2, }}, \cdots$, $P_{\text {RSEm }}$ which should be solved by means of the new goals and actions of rural policy, indirectly expressed through various types of knowledge: $\mathrm{K}_{\mathrm{RSEP} 1^{\prime}}, \mathrm{K}_{\mathrm{RSEP} 2^{\prime}} \ldots, \mathrm{K}_{\mathrm{RSEP} n^{\prime}}$ but only one situation for $\mathrm{D}_{\text {RSEN }} \mathrm{K}_{\text {RSEV }}$ and $\mathrm{C}_{\text {RSEB }}$. This can be schematically shown in Figure 2.3.1.

For the definition of the probability ( $\mathrm{Pr}$ ) for the formation of a socio-economic orientation (hereafter as $S_{R}$ ) which will revive rural development, it is necessary for each rural social or economic problem $\mathrm{P}_{\text {RSEi }}(i=1,2, \ldots, \mathrm{m})$, to define the probability $\mathbf{P}_{\mathbf{r}}\left(\mathrm{S}_{\mathrm{Ri}}\right)$; the sum of these probabilities is the indicator of the generation of new socio-economic orientation - I $\left(\mathrm{P}_{\mathrm{r}}\left(\mathrm{S}_{\mathrm{Ri}}\right)\right)$.

Under the presumption that the social and economic problems $\mathrm{P}_{\mathrm{r}}\left(\mathrm{S}_{\mathrm{Ri}}\right)$ are mutually independent, the probability of 
the formation of such a socio-economic orientation solving a i-th problem is:

$$
P_{r}\left(P_{R S}\right)=P_{r}\left(P_{R S i}\right) \times P_{r}\left(P_{\text {RSi }} D_{\text {RSEN }}\right) \times P_{r}\left(P_{\text {RSi }} K_{\text {RSEV }}\right) \times P_{r}\left(P_{\text {RSi }} C_{\text {RSEE }}\right)
$$

On this basis, the total probability of the formulation of socio-economic orientations which will solve rural social and economic problems is:

$P_{r}\left(P_{R S}\right)=\sum P_{r}\left(P_{R S i}\right) \times P_{r}\left(P_{\text {RSi }} D_{\text {RSEN }}\right) \times P_{r}\left(P_{\text {RSi }} K_{\text {RSEV }}\right) \times P_{r}\left(P_{\text {RSi }} C_{R S E B)}\right)$

$(i=1,2, \ldots, m)$

The appearance of the most probable socio-economic orientations that will overcome all rural social and economic problems comes from the simultaneous maximization of the value of equations (2.3.1.) and (2.3.2.). Greater priority is given to those socio-economic orientations which actively influence the solution to each particular problem.

\subsection{Application of the methodology in the concrete definition of general socio-economic orientations concerning the role of rural regions}

The basic purpose of the presented methodology presented here is to indicate thepossible factors to which it is possible to act. Namely, ilmplicitly, when analyzing factors influencing the formation of socio-economic orientation needed for the formulation and the realization of valid rural policies in the Western Balkans countries, we included conscious actions as instruments for findingdetermining proper solutions out. On the other hand, it is important to point out that the probability for of theone simultaneous positive action of all five specified factors and - on this basis - the establishment of new, (proper) socio-economic orientations, in the case of ythe Western Balkans, is low.

Within this context, the basic purpose of the methodology presented here is an attempt, based on its implementation, to identify those factors that need to be addressed. The ranking of their priorities should be done on the basis of criteria which exclude the factor of enforcement, i.e. compulsion, on the basis of the assumption that this entails a conscious process. In this way, the basic aim of actions will be automatically defined the precise identification of the issues and knowledge needed to arrive at a solution.

This access to the definition of socio-economic orientations in the selection of goals and actions of rural policy is cho- sen in accordance with the nature of political and economic interests as well as social and economic differences between rural and urban regions in the Western Balkans. The basic social and economic implication is that between the declared orientation that is necessary to stimulate rural development and the real socio-economic actions taken exists a large discrepancy. Although such a situation suggests that, in fact, the real political, institutional, social and economic structures and their basic analytic categories are realized in a form which does not permit the precise and clear realization of a proper rural policy, in our opinion, the limiting influence of this factor should not be analyzed in this manner. Because of an understandable fatigue that arises from societies in transition, its improvement is only possible over a long evolutionary process, assuming that it does eventually succeed in removing various obstacles to development.

This access, in itself, implicitly suggests one important limitation in conceiving rural policy. The rural policies in the Western Balkans cannot be based on rural structural funds, but should be based above all on "soft" elements, where solutions should be sought within the differences in the subjective standpoints of the participants (the rural population, political elite, economic elite, national institutions for the regulation of rural social and economic development, similar EU institutions for the same regulation, entrepreneurs, managers, etc.) and individual proposals.

On the other hand, the great similarity in the structure and substance of social and economic problems in typical rural regions (e.g. in the hilly - mountainous regions far away from actual development poles) in the Western Balkans should, on the basis of the methodology presented here and the exchange of experiences through its realization, provide for the formulation of new, proper socio-economic orientations.

In order to illustrate this, we will try to present the influence of international cooperation on the formation of new socio-economic orientations to address social and economic problems. We describe the example problem as the fact that "a rural economy in hilly - mountainous regions is not adapted to rational functioning under the conditions of a market economy." The purpose of the research performed can be summarized by the question "Which elements of institutional, social, economic and cultural structure should be changed for the sake of improved participation in European integration processes?" This study aimed to define the basic socio-economic orientations that would help solve this problem.

However, before the brief presentation of the potential role of international cooperation in developing a new socio- 
economic orientation for hilly - mountainous rural regions in the Western Balkans, it is necessary to first point out that their basic social and economic problems (and the knowledge required to overcome them) have a larger meaning. Their definition should result from the conscious analysis of a long term process covering the period before the inclusion of the Western Balkans in the modern capitalistic economy as well as the unsuccessful attempt to replace its previous socialist industrialization. Rural social and economic problems were caused above all by unsuccessful attempts at exploitation, including the unproductive implementation of European institutional, administrative, social and economic standards and values that scarred both the region's economy and demographics, and squandered its natural and manmade resources.

On the other hand, knowledge that should be applied to rural policies (based on the implementation of European institutional, social and economic standards and values) in the rural regions of the Western Balkans is generally of a practical, immediate nature.

It is necessary in this context to form a complex base of knowledge to overcome demographic and economic depression. This base should consist of (1) knowledge needed for the definition of new socio-economic orientations relating to the future roles of rural regions on the basis of the recognition of the essence of the long term processes of demographic, social and economic development and the creation of long-term, sustainable rural development and (2) knowledge needed to formulate exact goals and actions of rural policy with the end of eventually joining European integration processes. This should have a predominantly anti-cyclic character in order to interrupt negative economic and demographic trends within a short period of time and to reorient them to new, socially acceptable currents.

Figure 2.4.1 is a graphic illustration of the classification of chances for the appearance of new socio-economic orientations which would assist in overcoming the social and economic problem expressed above.

In Figure 2.4.1., the symbols used stand for the following:

$P_{i}\left(P_{R S E}\right)$ - the probability of overcoming rural social and economic problems in the rural, depressed, hilly - mountainous regions in of the Western Balkans.

$\mathrm{P}_{\mathrm{i}}\left(\mathrm{K}_{\mathrm{RSEP}}\right)$ - the probability of the formation of a base of knowledge for solving social and economic problems in rural, depressed, hilly - mountainous regions in the Western Balkans.

$P_{i}\left(D_{\text {RSEN }}\right)$ - the probability of structuring the development (social and economic) needs of rural regions, based on the use of natural and manmade resources in the rural regions of the Western Balkans.

$P_{i}\left(K_{R S E V}\right)$ - the probability of the formation of a structure of criteria expressed by the prevailing system of values in terms of new rural social, economic and cultural values which would help overcome demographic and economic depression in the rural regions of the Western Balkans.

$P_{i}\left(C_{\text {RSEB }}\right)$ - the probability of forming political, institutional, social and economic infrastructures in which the new rules of the game would be accepted (i.e., new social, economic and cultural values) and possibly reverse the demographic and economic depression in the rural regions of the Western Balkans ( $i=1$, Albania; $i=2$, Bosnia and Herzegovina; $i=3$, Croatia; $i=4$; Republic of Macedonia; $i=5$, Serbia and Montenegro).

On the basis of international cooperation, it is possible to realize the mutual substitution of the positive action of basic factors for the formulation of new socio-economic orientations if one keeps in mind that $\mathrm{P}_{\text {RSE' }}, \mathrm{K}_{\text {RSEV }}$ and $\mathrm{C}_{\text {RSEB }}$ are sufficient because of the large amount of knowledge $\left(\mathrm{K}_{\mathrm{RSEP}}\right)$ necessary to

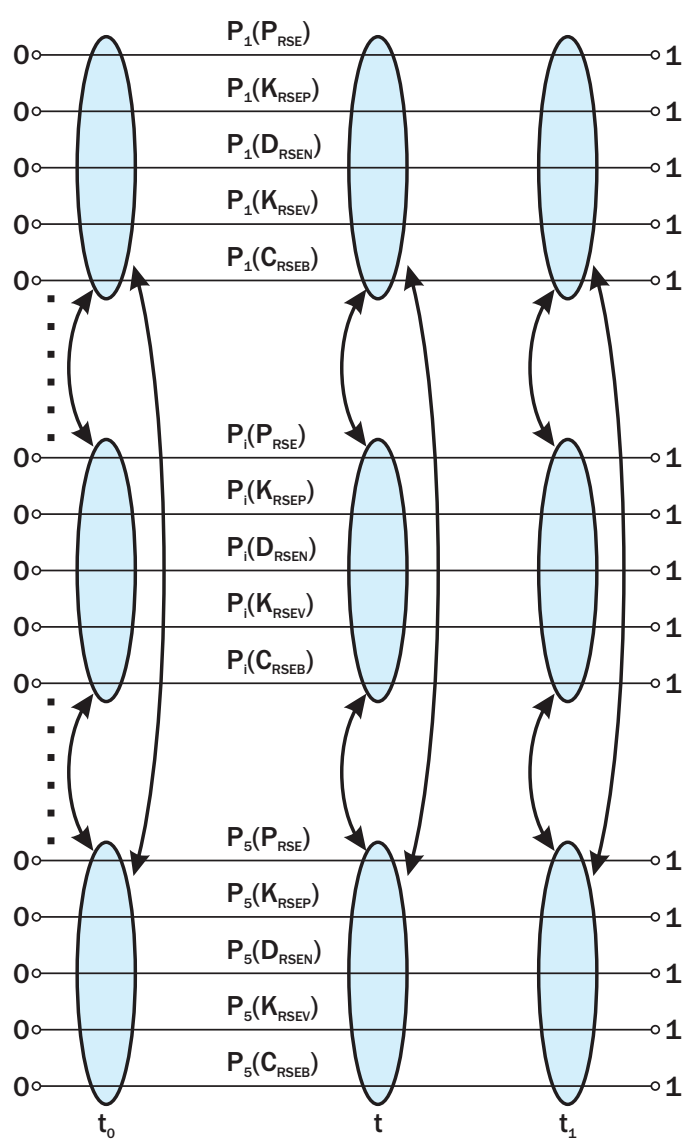

Figure 2.4.1.

Graphic illustration of the classification of chances for the appearance of a new socio-economic orientations concerning the role of rural regions in the Western Balkans 
define these new orientations (due to the exchange of expert knowledge between the countries of the Western Balkans) and the conditions' high degree of suitability for their implementation (due to the pressure of EU institutions on national political elites).

\subsection{Critical observation on the applied modeling methodology for the precise definition of the general rural socio-economic orientations for the future role of rural regions}

The basic feature of the methodology presented here for the definition of general socio-economic orientation as the basis for the precise and transparent definition of the goals and actions of rural development and their realization is its high precision based on the radical study of the experiences of countries with similar social and economic problems and historical heritages. For this reason the possible effective realization of this methodology should be based upon an adequate integration of the "learning" paradigm, which in turn would be based on a precisely and transparently defined set of goals and actions for rural development that acknowledges the necessary integration into Europe of the Western Balkans. Socio-economic orientation pertaining to the future role of rural regions in the Western Balkans should be the result of complex interactions composed of changes in the perceptions and reactions of political and economic elites, institutional changes and changes in the culture of rural businessmen and the population at large.

\section{Basic Methodological Conception of Modeling Rural Policies as Ill-structured Problems}

\subsection{Introduction}

This attempt to model rural policy within the frame of conscious action, and to identify this process of employing knowledge toward an ill-structured problem, necessitated a valid theoretical way of treating the ambiguities that result from subjective determinations of preference and the limitations and goals of private and public actors within a context to which a scientific analysis of formal structures was inapplicable.

Before attempting a detailed description of the model arguments will be presented to show why the precise and transparent definition of regional rural policy is necessary. The essence of the Western Balkans' rural socio-economic crisis comes from belated structural, social, cultural and economic adaptation. In this context the regional rural policy creates cooperation between countries to establish the goals and actions of national rural policies that would foster European Union integration.

National rural policy in this paper is considered a vague assemblage of goals and actions- social, economic and development policies that exceed all social, cultural and economic limits in advancing the development and cooperation between rural populations, entrepreneurs, managers, farms and small and medium rural enterprises.

This section will try to show the preliminary goals and actions of rural policy. Considering the actual level of external and internal ambiguities, we can say that the model for describing and evaluating the goals and actions of rural policy is an open model of inadequately clear structure, organization and dynamics.

One of the alternatives to the approach of describing and evaluating the goals and actions of rural policy is the use of the theory of multivariate planning based on a soft system combined with a multi-criteria compromise - choice. The high level of subjectivity and ambiguity in profiling rural policy does not diminish the value of this methodological approach, especially if the paradigm "learning" is incorporated to a larger extent into its formulation and evaluation, which (with the additional information from institutions and the praxis of rural development of the European Union), adds an analysis based on the expreiences of other governments of integration into the European Union. This should enable the realization of more effective reform.

\subsection{Application of the concept of a functional hierarchy of layers in modeling rural policies}

Since the basic problems in the definition of the attributes (the goals and actions) of rural policies in the Western Balkans countries we located in the domain ofare those of decision making, decisions, we proposed to installfor our in modelingmodeling an approach being the basedis onf the developed the concept of thea functional hierarchy of layers in decision making decisions (SchemFigure 3.2.1). This hierarchy is firmly based on natural principles because itand covers three basic aspects of decision making decisions under the conditions of incertitude: (1) the choice of strategy to be used in the process of problem solving,resolving. (2) the reduction and eventual removal of incertitude, and (3) looking for thea desirable di- 


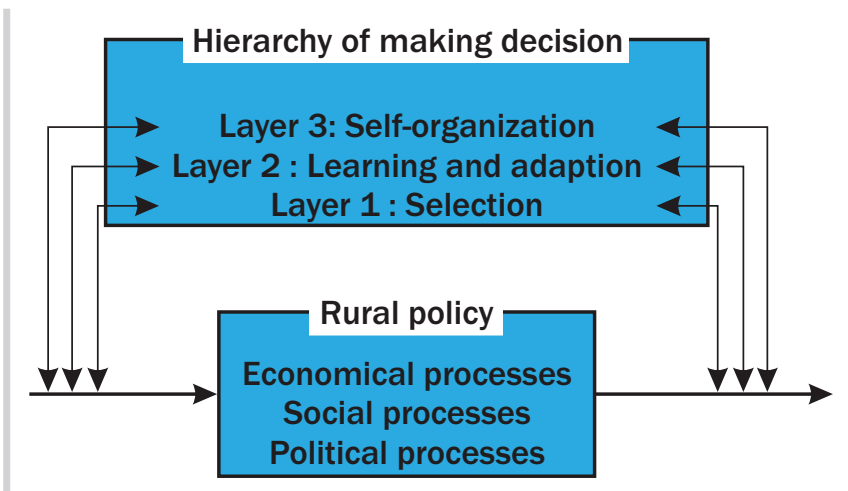

Figure 3.2.1.

Hierarchy of decision making by layers

rectioncourse of action under in advance of acceptable conditions.

The basic tasks of each layer are as follows:

1. Layer of selection - the selection of the course of action from an assortment of possibilities. On the basis of information from immediate sources and an algorithm defined by superordinate layers, the course of action is decided upon.

2. Layer of adaptation and learning - the reduction of incertitude by using paradigms such as competition, cooperation and learning specified by the superordinate self-organizing layer. The feature of adaptability allows the correction of parameters and the improvement of the procedure governing an action on the selected layer.

3. Self-organizing layer - evaluation of correctness and justification for resolutions and corrections.

On the basis of the general application of the principle of functional hierarchy in the formation of layers in decision making and the methodology of soft systems, the model of multivariate planning was selected for the analysis of the effects and costs of various combinations of attributes of rural policies in the Western Balkans.

\subsection{Multivariate planning (programming) and modeling of rural policies}

For describing and evaluating the goals and actions of rural policies we used a multivariate version of Checland's methodology for soft systems (Scheme 3.3.1.). This methodology is a seven-level analytical process thought the most appropriate system of learning for its use of nine mental processes: perception, comparison, assertion and desision making, which enables flexible problem solving.
In the first and second phases, it is necessary to gather information to locate the main difficulties in adaptation for rural societies and economies in the open market economy. Based on the entirety of our research, relevant standpoints were formed abstractly: "Rural societies and economies in the Western Balkans are incapable of funcioning in an open market environment. Are the elements of a country's economic, social and political organization and their interactions the main reasons for this problem?" Collected and systematically arranged knowledge should create a picture within which the following relevant topics require definition. 
1

Problem condition:

Revitalization of rural infrastructure on a local level and the choice of the model of providing supports from central public funds non-structured

Revitalization of rural infrastructure on a local level and the choice of the model of providing supports from central public funds

\section{6}

Basic limitations:

- Difference in rural development

- Incompatibile rural economic structure

- Inprecize elite reteptions and responses

- Undeveloped enterprenours and conservative and passive culture

- Anti-development oriented rural and agrarian polices

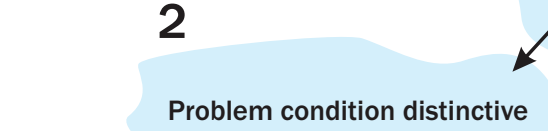

Real world

\section{Virtual world}

3

Initial definitions of relevant problems:

1. What should be done?

2. What this should be done?

3. Who should do this?

4. Who has a benefit and who suffer damage?

5. What limitations, which cannot be overcome are posed by surroundings?

\section{Criteria:}
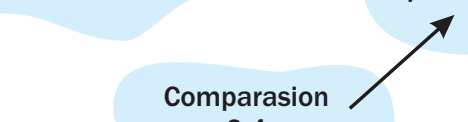

5

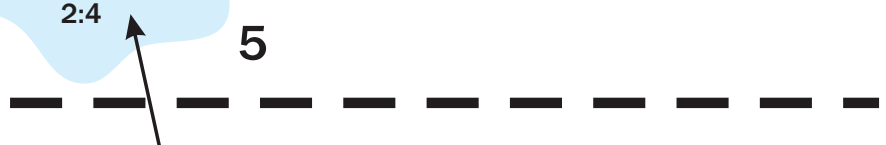
"A" U "B" U "C" U "D" U “E”

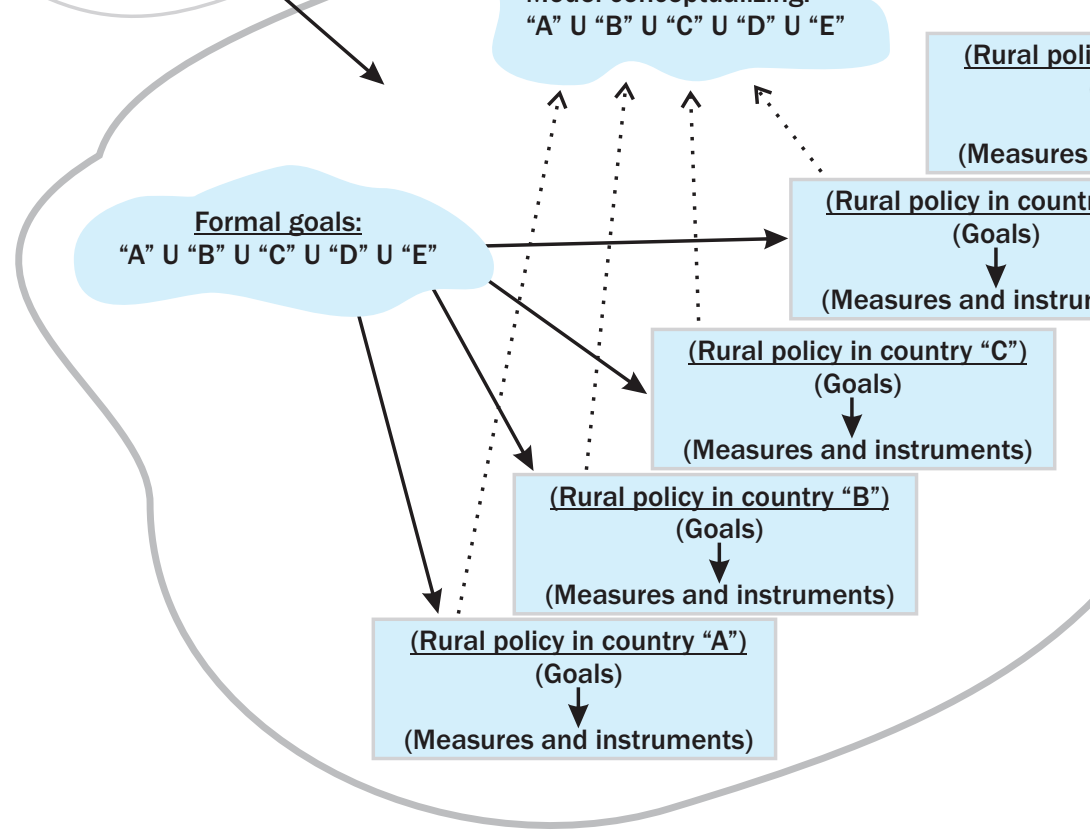

Model conceptualizing:

"A" U "B" U "C" U "D" U "E"

4

4

(Golicy in country " $\mathrm{E}$ ") (Goals)

$\downarrow$ instruments)

in country "D")

Figure 3.3.1.

Checkland's methodology for soft systems 


\section{Example: Revitalization of rural infrastructure on a local level and the choice of the model for providing support from central funds}

1. Problems - in the form of a vague assembly: \{The elite receptions and responses in relation to the role of rural society in development (unclear, imprecise and in essence negative for rural development), The political institutions (low credibility with regard to rural areas), the rural physical infrastructure (undeveloped, in ruin), the agrarian administrative infrastructure (undeveloped, without development strategy), the agrarian organisation and economic structure (lack of concurrence between internal and external markets, financial and technological ruin), financial system (undeveloped, disinterest in market behaviors), the rural enterprenuers and their managerial knowledge (scarce and archaic), the rural education system (poor quality, separated socially and culturally from the rural population), rural social problems (old age of the rural population, weak condition of the rural population, widespread and pronounced poverty\}

2. Actors - also in the form of a vague assembly: \{the rural population, rural enterprenuers, rural managers. the political elite, the economic elite, the central institutions for rural development, the local institutions for rural development, the institutions of the European Union for rural development, ...

3. Aggregates - also in the form of a vague assembly: \{the government administration for rural development, the local administration for rural development, public regulation, local regulation, national capital, local capital, the administration of the European Union, European regulation, ...

From the picture established, different topics arise, generating relevant perspectives in the form of a vague assembly:

$\{\text { Problems, Actors, Aggregates }\}_{\text {Western Balkans }}=\{\text { Problems, Actors, Aggregates }\}_{\text {Albania }}$

$\mathrm{U}\{\text { Problems, Actors, Aggregates }\}_{\text {Bosnia and Herregovina }} \cup\{\text { Problems, Actors, Aggregates }\}_{\text {Croatia }}$

U \{Problems, Actors, Aggregates $\}_{\text {Macedonia }} \cup\{\text { Problems, Actors, Aggregates }\}_{\text {Serbia and Montenegro }}$

On the basis of the totality of research presented in the form of a vague assembly \{Problems, Actors, Aggregates $\}_{\text {Western Balkans }}$ in the third phase of a vague assembly \{Abstract picture: The rural policy $>>$ to activate work and natural rural resources $->$ to restart stalled rural development $\rightarrow$ the structural adjustment of rural regions of the Western Balkans to European Union integration criteria -> rural infrastructure is formed giving the original definitions of the problem in the form of refined verbal statements. The basic goals in the third phase are to determine:

- What should be done?

- Why should it be done?

- Who should do it?

- Who should benefit, and who should suffer?

- What are the environmental limitations for each region and which are incapable of reform?

The content of our example, in a vague assembly \{Abstract picture: the rural policy -> to activate work and the use of natural rural resources -> to restart stalled rural development -> the structural adjustment of rural regions of the Western Balkans to European Union integration criteria -> the rural infrastructure\} shall be focused on:

1. The basic political and economic grouping and their ability for structural transformation of rural infrastructure and revitalization of development functions of the agricultural complex (modernizing of the rural physical infrastructure, agrarian administration, agriculture transformation, transformation of the finance system for servicing agriculture and the rural population, structural adjustment of the education system, development of rural social infrastructure, development of regional cooperation in the domain of agricultural and rural social policies. 
2. Internal and external factors of the surroundings that reinforce existing regional social and economic depression.

3. Determining potential actors ready and motivated to intensify development cooperation (the rural population, rural enterpreneurs, rural managers, European institutions, ...).

In the fourth stage, the transformation of the vague assembly \{abstract picture: the rural policy -> to activate work and the use of natural rural resources -> To restart stalled rural developmentl $->$ The structural adjustment of rural regions of the Western Balkans to European Union integration criteria -> the rural infrastructure into main activities is carried out in the form of the vague assembly \{Regional (rural) cohesion policy\}. Due to the abovementioned particularities concerning the structural adaptation of all rural infrastructure, it is necessary to create an abstract picture in the form of a vague assembly, with the parallel existence of several possible solutions:

Picture $« A »=\{\text { Albania: } \text { Rural policy }\}_{\text {Preparation for European Union integration }}$

Picture $« \mathrm{~B} »=\{$ Bosnia and Herzegovina: Rural policy $\}$

Picture $« C »=\{$ Croatia: Rural policy $\}$

Picture «D» = \{Macedonia: Rural policy

Picture «E»= \{Serbia and Montenego: Rural policy $\}$

This context includes:

$\{$ Rural policy: Goals $->$ Preparation of rural areas for European Union integration $\}=\{$ Albania: Measures and Instruments of Rural policy\} $\cup$ \{Bosnia and Herzegovina: Measures and Instruments of Rural policy\} $\cup$ \{Croatia: Measures and Instruments of Rural policy\} $\cup\{$ Macedonia: Measures and Instruments of Rural policy\} $\cup\{$ Serbia and Montenegro: Measures and Instruments of Rural policy\}

The final results may be modeled:

\{Rural policy: Goals -> Preparation of rural areas for European Union integration\}

with a structure comprising several parallel levels of realization of the national rural policy.

In the fifth stage, a comparison of the structured model:

\{Rural policy: Goals -> Preparation of rural areas for European Union integration\}

- in reality serves to forecast how the chosen structure of the model will behave in the future with the definition of \{Actors\} influencing the process of developing cooperation. In the context presented, the transformation of the vague assembly:

\{Multiateral programs for building the modern rural infrastructure of the Western Balkans\}

composed of the combination of measures and instruments of all national rural policy.

On the basis of its structure, formed by comparison, in the sixth stage it is necessary to perform the analysis of limitations (i. e. restrictions) as the final framework for changes. In the concrete example, the following restrictions have particular importance:

- Differences in rural economic and social development between regions in all Western Balkan countries.

- Incompatibile rural economic structure in all Western Balkan countries.

- Inprecise elite receptions and responses to the development of problems in the modern rural social infrastructures of all Western Balkan countries. 
- Undeveloped enterprenuers and a conservative and passive culture in all Western Balkan countries.

- Incompetent rural and agrarian administrations in all Western Balkan countries.

- $\quad$ Anti-development oriented rural and agrarian policies in all Western Balkan countries.

Accordingly the following vague assembly is formed:

$\{$ Restriction $\}=\{$ Difference in rural development $\} \cup\{$ Incompatibile rural economic structure $\} \cup\{$ Inprecise elite receptions and responses $\} \cup$ \{Undeveloped enterprenuers and a conservative and passive culture $\cup$ \{Incompetent rural and agrarian administrations $\} \cup\{$ Anti-development oriented rural and agrarian policies $\}$

On this basis, a comparison between desirable and feasible changes can be carried out:

$\{$ Multiateral programs for building the modern rural infrastructure in Western Balkan countries $\}$ \{Restriction $\}=\{$ Multiateral programs for building modern rural infrastructure in Western Balkan countries\}

The phase of implementation (seventh phase), based upon the large spectrum of measures and instruments of social, economic and development policies adapted to different rural structures (rural and agrarian administrations, rural physical infrastructure, agriculture, financial systems for servicing the agriculture and rural populations, the education system, rural small and medium enterprises, ...) in all Western Balkan countries.

The methodology presented here is based on the procedure of system observation to discover "What are the best ways to solve the observed problem?" - in which a large number of actors with partial observations and truths, and fusibile analysis are incorporated. The integration of these-

$\{$ Actors, Aggregates\} -> \{Goals: The effecive integration of rural areas into the European Union\} -> $\{$ Measurement and mechanism of rural policy\}

enables one, through a learning process, to conduct a more robust and flexible structuring of content for the realization of rural policies in the social, economic and development sectors.

\subsection{Critical observation on the applied modeling methodology of rural policies}

The methodological conception of the definition of rural policy attributes presented here provides: (1) an improvement to the systematic approach in researching basic causes and alternative models when resolving rural social and economic problems, (2) the introduction and application of models which, regardless of an open and insufficiently precise structure, may be used for the definition and correction of goals and activities in the realization of desirable rural changes and (3) the creation of a culture of behavior for actors wherein an openness towards their surroundings and efforts to overcome conflict between subjects have priority.

The methodology presented here is based on the suppositions that: (1) a culture of participation exists and (2) differences in individual perceptions relating to key phenomena in the social sphere, differences in the opinions of experts and other participants may be harmonized in a natural way, without compulsion.
There also emerge basic problems in the proposed methodology because it is impossible to specify: (1) "Who should be included?" and (2) "How much participation?"

The advantage of the application of the proposed methodology should be sought in the sphere of the system of didactics of all relevant participants. The presented methodology is based on the systematic observation: "What is the best mode of resolving this problem?" wherein a series of feasibility analyses and actions are included on one hand and a large circle of actors with partial views and subjective convictions on the other. The integration of views and approaches on the principle of step by step in the form of vague outlines and transformations in the chain \{Actors, Aggregates\} ---> \{Goals of rural policy\}---> \{Measures and instruments of rural policy\} makes possible the process of learning - which, at this moment, has priority with regard to the paradigm of optimalization expressed as Cost --$>$ Results. 


\section{Decision Making in Modeling the Attributes of Rural Policies}

\subsection{Introduction}

One Ssuggested technique for modelingmodeling the attributes of rural policies is based on information and decisions in the form of verbal statements. In accordance with itthis, a methodology developed on a "fuzzy system" theory iwas chosen for analyzing the decision making problems in modelingmodeling the rural policyies attributes. The lintroduction of a fuzzy system is connected to the theory of fuzzy (spreading) assemblassemblies, in order to provide a standard mathematical treatment of imprecisenession, which is characteristic forof the linguistic approach toin modelingmodeling.

The fuzzy system connects the inputs of vague assemblies with output assemblies. Fuzzy systems are determined through three phases: (1) determination of variables, (2) definition of the values of variables and (3) setting rules for fuzziness.

How does the fuzzy system work? All the rules are located within the fuzzy system. These rules are activated in parallel and partially. Each rule has the shape: If $A$ then $B$. The input value $x$ activates the $A$ part of each rule to some degree. It gives us then part $B$ of the rule to some degree. As a result we get a fuzzy approximate average. That average is the center of mass or center which clarifies the vague assembly.

Fuzzy logic enables us to make decisions based on estimated values. These values are based on incomplete information. The valuable conclusions thereof are presented in a linguistic form. Of course, decisions can be incorrect and then cause the need for corrective actions based on additional available information.

The implementation of fuzzy logics into the rural policy model (in the chosen example: the reconstruction of rural infrastructure and its financial model on a local level) is based on the following key feed back relating to subsystems:

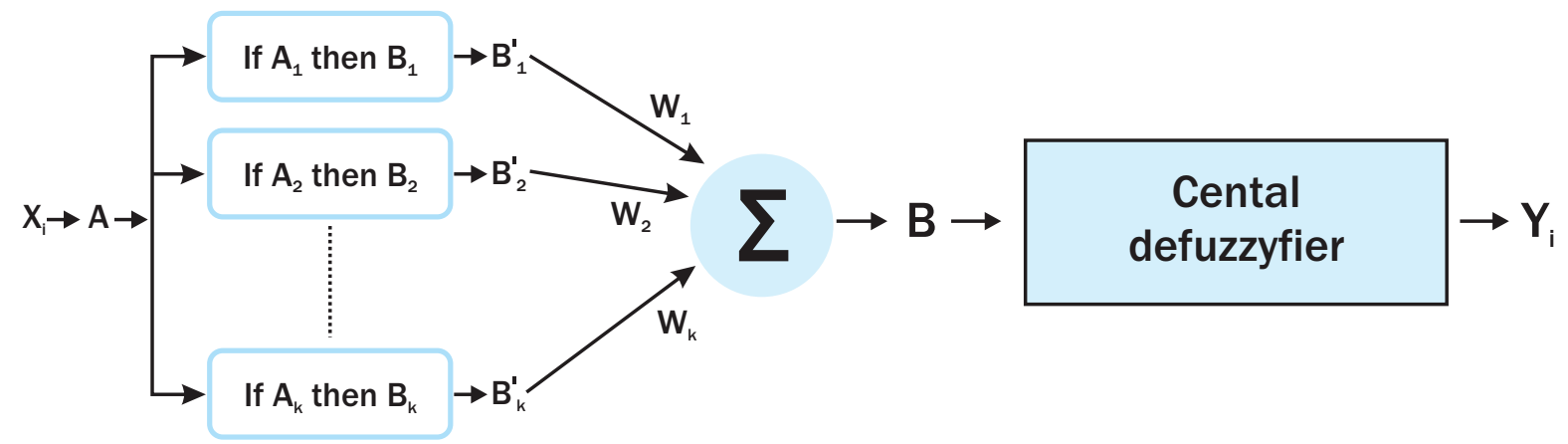

Figure 4.1.1.

"Conclusion" scheme of a fuzzy system

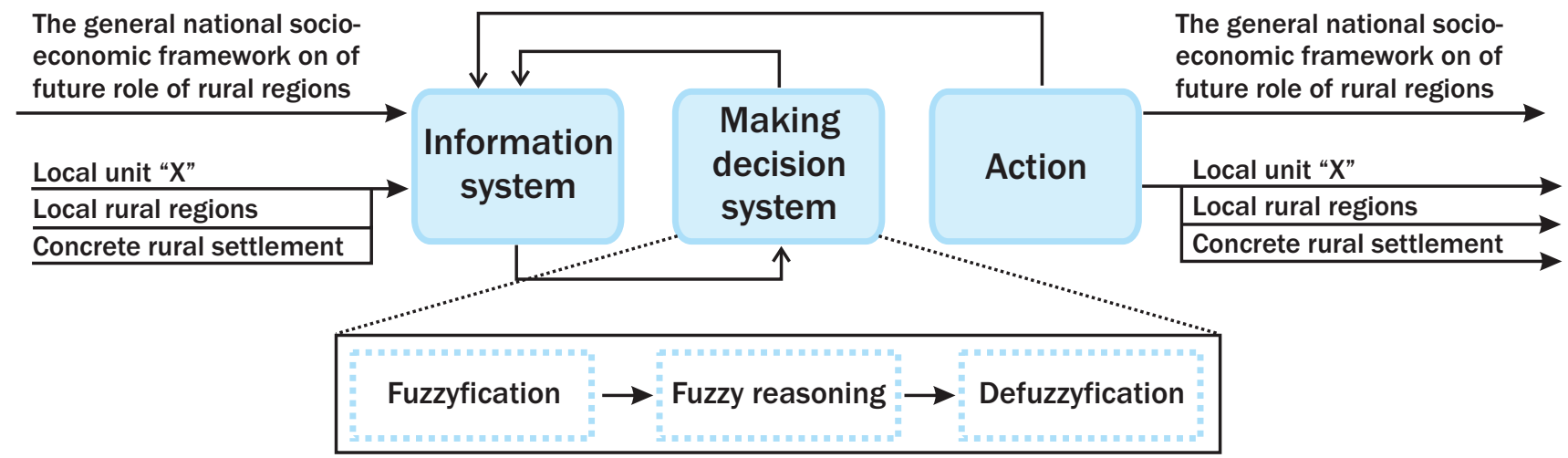

Figure 4.1.2.

Fuzzy logics implementation methodology 


\subsection{The methodology for making multi-criteria decisions and the problem of overcoming indefiniteness in modeling rural policies}

The basic idea behind structuring a fuzzy model for making multi-criteria decisions in order to overcome the problems of indefiniteness in modeling rural policies is based on its application to the second, third and fifth phases of Chekland's soft system methodology in outlining the goals and actions of rural policy.

In order to illustrate the suggested methodology, we will use the example of the program: REVITALIZATION OF RURAL INFRASTRUCTURE ON A LOCAL LEVEL AND THE CHOICE OF THE MODEL FOR PROVIDING SUPPORT FROM CENTRAL FUNDS. We will present the following decision making process: "Is it necessary to selectively provide additional means from central public funds for the participation of the cost of revitalization of rural infrastructure for a local unit " $X$ "?"We introduce fuzzy variables, described verbally (Table 4.2.1.):
Because the variables in the above table - from which any decisions must be made - are described verbally their values are determined to be relative values or to varying degrees based on experts' estimates. Hence, the variables used in deciding on the actions for achieving determined goals are characterized by: (1) polysemia, due to the possibility of different interpretations of the described phenomena, (2) lack of coordination and (3) meagerness of content. All of this is due to the problems in the unique determination of different content and mistakes in the definition of limits between different phenomena.

Decision making is based on a fuzzy reasoning algorithm (Figure 4.2.1)

\begin{tabular}{|c|}
\hline $\begin{array}{l}\text { Medium-term changes in the dynamics of } \\
\text { development of rural regions in the local unit " } X \text { ": }\end{array}$ \\
\hline $\begin{array}{l}\text { Long-term changes in the dynamics of development } \\
\text { of rural regions in the local unit " } X \text { ": }\end{array}$ \\
\hline INTERNAL FACTORS \\
\hline $\begin{array}{l}\text { National standardization of minimal infrastructural } \\
\text { services being ensured from public means for rural } \\
\text { regions and population: }\end{array}$ \\
\hline $\begin{array}{l}\text { Total public revenues from local economy } \\
\text { and population: }\end{array}$ \\
\hline $\begin{array}{l}\text { Public revenues from local rural economy and } \\
\text { rural population: }\end{array}$ \\
\hline Total local public expences: \\
\hline $\begin{array}{l}\text { Expencses from from central funds (for rural development, } \\
\text { for subventions, for subventins for rural economy, for education } \\
\text { of rural population,for social protection of rural population) } \\
\text { - in rural regions in the local unit " } X \text { ": }\end{array}$ \\
\hline
\end{tabular}

Local public expencses in rural regions (for rural development, for subventions for rural economy, for education of rural population, for social protection of rural population):

External financingement (non-governmental organizations, international help):

IMPLEMENTATION

\section{Corruption:}

Administrative compulsion:

Ethical (political) compulsion:

Expertnessise (public managers and administrations): development, status quo, reduction

development, status quo, reduction

development, status quo, reduction

favorable, neutral, unfavorable

high, average, low

high, average, low

high, average, low

high, average, low

high, average, low

high, average, low

high, average, low

high, average, low

enormous, significant, minor

high, average, low

aggressive, moderate, passive

high, average, low

Table 4.2.1. 


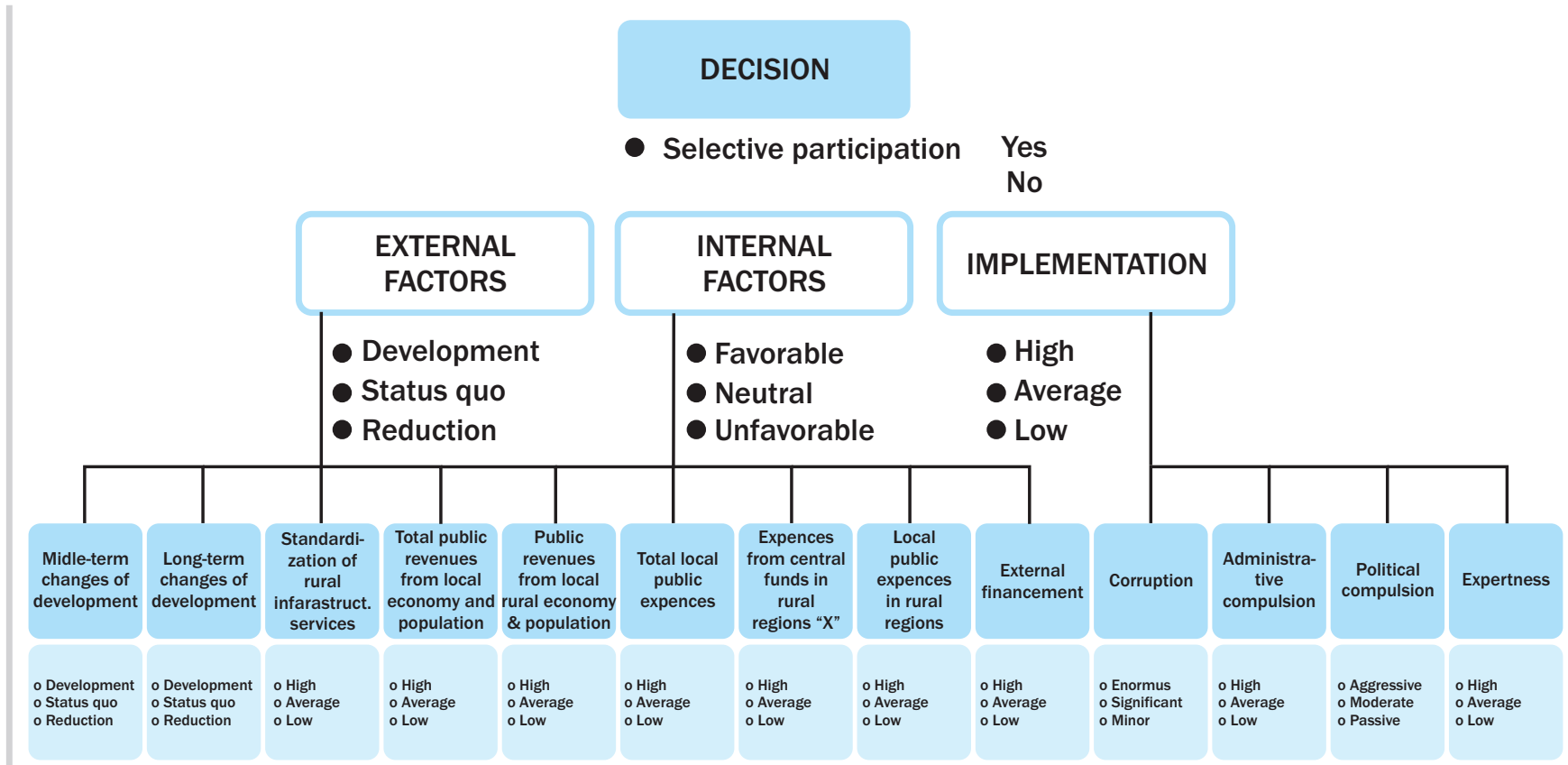

Figure 4.2.1.

Algorithm of fuzzy reasoning

\begin{tabular}{|c|c|c|c|}
\hline & & \\
\hline
\end{tabular}

Table 4.2.2.
Input values for a concrete local milieu in linguistic forms are "fuzzified", associated with numerical values and the degree of pertinence of output variables: EXTERNAL FACTORS, INTERNAL FACTORS, IMPLEMENTATION are ascertained with the minimum degree of pertinence.

The output values are defined by rules having different values based on experts' estimates, with different degrees of pertinence. "Defuzzification" gives us concrete numerical values for output variables, while the final output decision, selective provision of additional means from central public funds for the participation of the costs of revitalization of rural infrastructure for a local unit " $X$ ", yes or no, we obtain on the basis of the IF THEN rule:

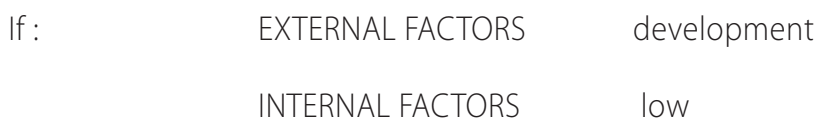

Then the

decision is: IMPLEMENTATION yes 


\subsection{Critical observations on applied multi-criteria decision methodology in modeling the attributes of rural policies}

The suggested multi-criteria methodology of decision making is characterized by the high level of subjectivity and indefiniteness caused by experts' estimates. However, in combination with the abovementioned hierarchy of decision making methodology through layers (specifically layers for adaptation and learning), it is possible to increase the objective degree of making decision by involving three paradigms: (1) Competition, as the frame for individual and collective decisions, (2) Cooperation, as the main way for providing rare resources (in this case resources and institutions for implementing social policy actions) and (3) Learning, in order to provide in due time an effective decision based on additional information resulting from analysis of the effects of social policies attributes, mainly from an "ex ante" outlined assembly of actions.

\section{Conclusion}

Application of the presented methodology for the modeling of rural development and its realization in two steps serves as a basis for the combination of different methodological concepts with different levels of precision. To gradually make volume and time dimensions narrower as common research measures would provide a valid way for treating ambiguities and fuzziness which are inherent in subjective determination and the preferences and limitations for actors collaborating within an environment lacking science, practice or tradition with respect to formal structures. Their basic characteristic is a high level of precision in making fundamental social and economic decisions on the one hand, and high subjectivity in determining the concrete content of problems to make decisions on the other. The achieved results can be used for a satisfactory open version of rural policy. Its effective realization depends on the adequate integration of the paradigm learning with a precise set of goals along with passive and unaddressed measures and mechanisms of rural policy. A clearly defined course of action and the development of cooperation should result from a complex interaction between institutional changes, changes among the elite and changes in the cultural behavior of the rural population and its enterprenuers.

\section{References}

Adzic, S. and Sedlak, O. (1997), System Approach to the Developing Policies as Ill-Structured Problem, PC 120 PAMM, Balatonalmady.

Adzic, S. and Sedlak, O. (1997), The Model of multicriteria decision making within National Sector Policies, 4th Balkan Conference on Operational Research,Thessalonici.

Adžić, S. and Adžić, J. (2004), Multi-Criterion Decision Modeling within Rural Social Policy - The Case of South-East Europe, "Sixt International Conference on Social Science Methodology - RC 33 Logic \& Methodology”, Amstedam.

Bowles, J. B. and Palaez, C. E.(1995), Application of fuzzy logic to reliability engineering, in Proceedings of the IEEE, Vol. 83 No3.

Checkland, P. B. and Sholes, I. (1990), Soft System Methodology in Action, Wiley, Chichester.

Disley, F. (1994) Irrationality of decision making, in OR Newsletter, March, 1994.

Sprague, R. and Watson, H. (1989), Decision Support Systems - putting theory into practice, Prentice Hall, London-Sidney-Toronto,

Zadeh, L. A. (1994), Fuzzy logic, neural networks and soft computing, ACM, Vol. 37, No 3, 1994.

Wilson, B. (1991), Systems: Concepts, methodologies and application, Wiley, Chichester. 